\title{
MVsim: a toolset for quantifying and designing multivalent interactions
}

Bence Bruncsics ${ }^{\mathrm{a},{ }^{*}}$, Wesley J. Errington ${ }^{\mathrm{b},{ }^{,}}$, and Casim A. Sarkar ${ }^{\mathrm{b}, 1}$

a Department of Measurement and Information Systems, Budapest University of

Technology and Economics, Budapest $\mathrm{H}-1111$, Hungary

${ }^{b}$ Department of Biomedical Engineering, University of Minnesota, Minneapolis, MN 55455-0215, USA

* Contributed equally

1 To whom correspondence may be addressed: csarkar@umn.edu 


\section{Abstract}

Arising through multiple binding elements, multivalency can specify the avidity, duration, cooperativity, and selectivity of biomolecular interactions, but quantitative prediction and design of these properties has remained challenging. Here we present MVsim, an application suite built around a configurational network model of multivalency to facilitate the quantification, design, and mechanistic evaluation of multivalent binding phenomena through a simple graphical user interface. To demonstrate the utility and versatility of MVsim, we first show that both monospecific and multispecific multivalent ligand-receptor interactions, with their noncanonical binding kinetics, can be accurately simulated. We then quantitatively predict the ultrasensitivity and performance of multivalent-encoded protein logic gates, evaluate the inherent programmability of multispecificity for selective receptor targeting, and extract rate constants of conformational switching for the SARSCoV-2 spike protein and model its binding to ACE2 as well as multivalent inhibitors of this interaction. MVsim is freely available at https://sarkarlab.github.io/MVsim/.

Keywords: multivalency, binding simulation, protein design resource, kinetic modeling, surface plasmon resonance

\section{Main}

Multivalent interactions are fundamental building blocks of supramolecular systems. Deriving from multiple binding elements within sets of interacting molecules, multivalency is used to regulate intracellular compartmentalization ${ }^{1-5}$, high-avidity interactions ${ }^{6-9}$, ultrasensitivity ${ }^{10}$, and dynamics and selectivity of molecular recognition ${ }^{11-13}$. The expansive utility of multivalency has driven multiple computational approaches to describe aspects of multivalent interactions ${ }^{14-22}$. However, as the molecular systems of interest and synthetic design ambitions become increasingly manifold and complex, frameworks are required that are extensible across the multi-parameter landscape and that can be furthered into interactive tools for design and quantification. We previously developed a conceptualization of multivalency that described the noncanonical 
signatures of multivalent receptor-ligand interactions as the flux through an interconnected network of configurational microstates ${ }^{23}$. This approach provided highlyresolved mechanistic insights into the dynamical events that underlie simple multivalent interactions and indicated a means with which to extend existing experimental techniques - such as surface plasmon resonance (SPR) - to macromolecular systems previously beyond the scope of quantitative analysis due to their complexity and heterogeneity ${ }^{24,25}$. However, this conceptual framework was limited to monospecific multivalent interactions between proteins of certain topologies and was also not practically implementable to quickly analyze and design a wide range of molecular systems ${ }^{26-29}$.

Here, we have developed a new and expanded computational method based on the original conceptual framework, which we present as MVsim, an interactive toolset with a simple graphical user interface (GUI) for the design, prediction, multi-dimensional parameter exploration, and quantification of multivalent binding phenomena. MVsim enables users to simulate multivalent binding through an expansive implementation of configurational multivalent networks within the MATLAB software environment ${ }^{30}$. With user-specified kinetic, topological, and structural parameters, MVsim simulates the conformational dynamics and binding responses for multicomponent systems of multidomain, multispecific, and multimeric interacting biomolecules. Further, MVsim synthesizes its outputs as sets of interactive kinetic traces to facilitate visualization, inspection, quantification, curve fitting, and experimental implementation of the structureactivity relationships and the information-coding intrinsic to multivalency.

We first validate the ability of MVsim to accurately simulate both monospecific and multispecific protein-protein interactions, the latter of which was not possible in our initial model of multivalency ${ }^{23}$. To then demonstrate application of $M V$ sim, we use experimentguided modeling to quantify switch-like signaling of synthetically-designed systems ${ }^{31}$, uncover design rules and predict the response dynamics of multivalent logic gates ${ }^{31}$, and leverage multispecific ligand architectures to enable selective receptor targeting for therapeutic development ${ }^{10}$. Further, we apply MVsim toward the inspection and quantification of viral spike protein dynamics. At present, nowhere is the importance of 
multivalency more assertively illustrated than with the mechanics of infection and therapeutic targeting of the configurationally dynamic, trimeric SARS-CoV-2 $S$ protein $^{32,33}$, its dimeric ACE2 receptor ${ }^{34,35}$, and a growing library of designed multivalent and multispecific neutralizing inhibitors ${ }^{36-40}$. Here, we use MVsim to derive an effective concentration for the ACE2 interaction, quantify intramolecular rate constants of SARSCoV-2 S protein receptor binding domain (RBD) conformational switching that enable host cell engagement, and probe the consequences of variants with altered conformational stability of the $S$ protein. This series of multivalent and multi-ligand simulations served as an intuitive means to quantify the relationship between macromolecular topology and SARS-CoV-2 S protein response dynamics, infectivity, and refined approaches for therapeutic targeting.

In sum, MVsim offers an intuitive and easy-to-implement molecular design toolset, bringing enhanced quantification and predictive design of multicomponent and multivalent systems to protein engineering, molecular and cellular systems analysis, and therapeutic design.

\section{Results}

\section{Development of MVsim}

MVsim is a multivalent interaction toolset built upon our configurational microstate network model ${ }^{23}$, which expanded upon prior modeling efforts in the literature by explicitly treating multivalency as a dynamic ensemble of binding configurations driven through local, topology-derived effective concentrations. MVsim represents a reconceptualization and application of the initial, more limited network model to now provide mechanistic descriptions of an array of biologically and therapeutically relevant multivalent systems and to quantitatively predict binding responses and conformational dynamics across a breadth of parameter space $31,37,40$. 
The creation of the MVsim toolset translated the fundamental concepts of the network model into MATLAB coding environment through a series of implementations that represent significant advances in its ability to easily simulate a broad range of multivalent interactions. First, to describe a user-specified multi-ligand, multivalent interaction system, we developed a rule-based modeling routine to automatically enumerate all possible binding microstates and configuration transitions between them to generate a descriptive kinetic rate model (Extended Methods, Supplementary Information). For example, extended to its furthest, $M V$ sim simulates competitive interaction among three topologically-varied trivalent ligands for a trivalent receptor, described with a system of 1,538 differential rate equations.

Second, MVsim effectively and rapidly parameterizes the system of rate equations with computed topology-derived first-order rate constants of association. Here, MVsim uses dimensionally-reduced polar coordinate integrations of the molecular interaction volumes. With this approach, the frequency of all pairwise combinations of multivalent interaction between a ligand and receptor binding domain are calculated with joint probability density functions to yield a set of effective concentrations. This routine enables efficient calculations to be performed with high spatial resolution for nanoscale and mesoscale multivalent species with domain diameters, linkages, and persistence lengths exceeding $1000 \AA$.

Third, MVsim has an extensive multiparameter description of the molecular multivalent landscape that allows for zero-fit prediction of the response dynamics of fullyparameterized systems where experimental multivalent data is absent. Conversely, MVsim enables parameter estimation for topologically under-characterized systems where multivalent binding kinetics have been measured. MVsim facilitates quantification of multivalent binding responses in terms of effective rate constants of association $\left(k_{o n}^{e f f}\right)$ and dissociation $\left(k_{o f f}^{e f f}\right)$, equilibrium binding constants $\left(K_{D}^{e f f}\right)$, competitive inhibitor potency $\left(I C_{50}\right)$, and Hill coefficients describing ultrasensitive switch-like behavior. 
Interfacing MVsim with MATLAB's app design environment enabled creation of a tabbed GUI to guide the specification of biologically important manifestations of multivalency via multiparameter inputs in MVsim. The MVsim GUI enables full input parameterization of the domain and linkage topologies of the ligand(s) (Fig. 1a) and receptor (Fig. 1c), the monovalent kinetics between each pairwise combination of ligand-receptor binding domains (Fig. 1a), the temporal ligand concentration dynamics (Fig. 1b), and the set of parameters that govern SPR and related kinetic studies, including the association and dissociation times, flow rate, and level of immobilized receptor. Additionally, for instances where detailed topological information is known, MVsim allows users to directly input effective ligand concentrations and end-to-end probability density functions for the multivalent system of interest (Fig. S1). Finally, once a multivalent design has been input, the user can initiate MVsim and subsequently survey a range of non-topological parameter variants in quick succession (Fig. 1d).

\section{Simulation outputs}

Following the initiation of a simulation, MVsim provides users a variety of means to visualize, interact with, and export the simulated response kinetics. Most simply, the simulation results are displayed within the output field as an interactive plot of binding response signal as a function of the specified association and dissociation time (Fig. 2). Here, users can choose between two graphical outputs of the response kinetics. First, as is typical of experimental binding kinetic data, a plot of a user-specified ligand concentration is displayed (Fig. 2a). Alternatively, users can select a plot of all composite microstate configurations underlying the response signal (Fig. 2b), binned according to valency class (Fig. 2c) or observe the competitive binding dynamics among multiple ligands (Fig. 2d). MVsim additionally enables users to visualize the dynamic evolution of the microstate network via an interactive map (Fig. 2e) and to export the response kinetics as a set of tab-delineated text files to facilitate deeper analysis through offline plotting and curve fitting, and through microstate network analysis within the Cytoscape software environment ${ }^{41}$. MVsim additionally enables users to directly inspect both the computed 
probability density functions and effective concentrations (Fig. S2).

\section{Assessing performance against experimental model systems}

To evaluate the accuracy of simulating complex topologies, MVsim was used to predict the binding response dynamics of our previously constructed monospecific multivalent interactions and two new experimental systems (Fig. 3). In agreement with our previous studies of monospecific interactions, MVsim predicts monospecific multivalent binding (Fig. 3b,c) but now also shows improved sensitivity to the topological constraints that can impede certain configurations, such as those that require contorted twisting of interdomain linkages. We further used MVsim to make new predictions in both multispecific ligand and multi-ligand interaction systems. In the first validation, a multispecific receptor-ligand architecture was designed using two sets of protein-protein interaction domains (Fig. 3a). Experimentally determined monovalent kinetic rate constants and structure-derived molecular topologies were used to parameterize the model (Fig. 3a,d) and generate a simulated dataset (Fig. 3e). Comparing simulation with the corresponding SPR dataset (Fig. 3f) demonstrates a good agreement with regard to the ability of MVsim to predict a priori the magnitude and multiphasic character of the experimental binding responses. Further, MVsim provides mechanistic explanations for these binding responses, showing, for example, the contribution of high-stoichiometric configurations to the microstate ensemble driven by the use of rigid, $\alpha$-helical linkers (Fig. S3a-c).

As a second model-experiment validation, a fully parameterized, dual ligand interaction system was constructed (Fig. 3g). Again, comparing the simulated binding responses (Fig. 3h) to the corresponding experimental SPR data (Fig. 3i) shows good agreement between MVsim and experiment in the characteristic multiphasic appearance of both the association and dissociation phases of the interaction. Moreover, beyond simply predicting the overall kinetics of the system, MVsim provides insights into the mechanics of multiple multivalent and multispecific ligands competing for a receptor, and attributes these molecular properties back to the macroscopically observable features of the 
multiphasic binding responses. Here, for example, MVsim quantitatively captures how effective rate constants of dissociation can be dictated by valency and can be used to effect temporal ordering of interactions between a rapidly, but more transiently, binding monovalent ligand and a slower, but more avid, multivalent ligand (Fig. 3d-f).

\section{Applications to multivalent system design and quantification}

MVsim was established to both guide the design and implementation of multivalent properties and to facilitate parameter estimation for existing and incompletely characterized natural and synthetic multivalent systems. Here, the model's lack of reliance upon fitted parameters enables MVsim to better describe the additive, competitive, and cooperative relationships implicit between kinetic, topological and valency parameters and to apply these to the quantification of multivalent properties, such as effective concentration, avidity, and binding selectivity. To evaluate the performance of MVsim as a molecular design and quantification tool, we assessed its ability to design and predict binding response dynamics in four different instances and applications of multivalency.

MVsim predicts ultrasensitive behavior in engineered protein switches and logic gates

The effective concentration that drives multivalent binding gives these systems the inherent ability to produce nonlinear input/output response dynamics. It has been previously demonstrated, for example, that ultrasensitive toggling can be driven through the introduction of monovalent counterparts into a multivalent system ${ }^{31}$. Dueber et al. showed that cooperative competitive dissociation of multivalent protein-protein complexes effects switch-like transitions that can be leveraged to control the fractional saturation of receptor-ligand interactions and enzymatic activity. Here, we apply MVsim to study the activation dynamics of engineered bivalent and trivalent protein switches and identify critical parameters for optimal system performance. MVsim quantitatively predicts the relationship between the valency of the system and the magnitude of its cooperative 
transition to an active state (Fig. 4a). The functional range of multivalent switches can be extended through the incorporation of multispecific interactions. This design approach enables the creation of AND logic gates in which a switch response is elicited only by a programmed combination of molecular inputs. Using MVsim to model this system recapitulates the experimental three-input response (Fig. 4b) and reveals potential sources of erroneous activation in the design (Fig. S4). It also identifies the importance of minimizing steric imposition between intramolecular interactions, closely matching multispecific binding strengths across domains within the multi-domain construct, and ensuring the concentrations of the monovalent agonists required to facilitate dynamic switching are compatible with applications in cells and organisms (Fig. S4).

MVsim informs the use of multispecificity for molecular recognition and therapeutic targeting

Multispecificity is a potent molecular design element that is widely used in drug discovery and cell engineering. By leveraging two or more distinct binding epitopes, multispecific interactions are employed to engineer highly avid and selective molecular recognition for use in such applications as bispecific therapeutic antibodies ${ }^{10,27}$ and chimeric antigen receptor $\mathrm{T}$ cells ${ }^{42}$. Multi-site recognition additionally enables higher-order information processing, allowing these multispecific systems to generate differential outputs to varying combinations of inputs ${ }^{11,12}$.

Because the network model of multivalency computes multivalent binding as the cooperative sum of its composite interactions, MVsim is well-suited to the study of multipartite interactions. Here, we explore two important applications of engineered multispecificity.

First, MVsim examines the information-coding capacity of multispecific interactions to effect temporal ordering in a model regulatory system consisting of two kinases and a phosphatase (the 'ligands') that can consecutively engage a common signaling hub (the 'receptor') despite all three enzymes being simultaneously introduced. Here, exploration 
of the simulated parameter space revealed a design that enables serial binding events by exploiting the cumulative effects of concurrent binding afforded by multispecificity, the cooperative, competitive binding of multi-ligand dynamics, and the generation of effective dissociation rate constants via multivalency (Fig. 4c). Together, appending these multispecific interaction domains to the simulated regulatory system effectively creates a molecularly encoded program with biochemical and biophysical properties that specify the orderly progression of multi-ligand binding to enhancing system performance or specificity.

Second, multispecific interactions can be designed that maximally exploit any degree of variation in the type and number of surface receptors and antigens within a population for the purposes of selective targeting ${ }^{10}$. In this regard, we directed MVsim to address a design question: given a population of three distinct types of antigenic cell surfaces, what are the optimal ligand designs that can singly, doubly, and triply interrogate the population? MVsim demonstrates that the composition of the target receptor serves as a generally useful guide for ligand design, as seen, for example, in Fig. $4 \mathrm{~d}$ in the relative selectivites of mono-, bi-, and trispecific Ligands 1, 3, and 7, respectively, for Receptor 3. Moreover, selective recognition can be further maximized using designed linkages that leverage the spatial proximity between receptor target surfaces; in Fig. 4d, Ligand 2b (rigid linkage) has greater selectivity than Ligand 2a (flexible linkage) for Receptor 2.

MVsim models the multivalency and avidity of SARS-CoV-2 S protein interactions

At present, one of the most prominent and consequential displays of multivalent binding surrounds the surface spike (S protein) of SARS-CoV-2. The S protein is a sophisticated, conformationally activated molecular system that mediates the selective recognition of target cells and generates the driving force needed to overcome the energy barrier of membrane fusion, thus enabling viral entry into the host ${ }^{32,33}$. The multimeric and multivalent configuration of the $\mathrm{S}$ protein is central to these functions ${ }^{43}$. Trimeric assembly serves to stabilize the $S$ protein against erroneous fusogenic conformational changes, 
establish allosteric control, and potentially present multiple receptor binding domains (RBDs) that bind multivalently with a host cell surface populated with dimeric ACE2 receptor proteins ${ }^{43}$. In response to these natural displays of multivalency, this same principle has been mobilized in therapeutic designs intended to neutralize, inhibit, or otherwise uncouple the structure-activity relationship of the $S$ protein ${ }^{35-40}$.

Despite the significantly more complex multivalent architecture of the S protein compared with our previously described applications, MVsim can be effectively parameterized to model and quantify critical structural properties of the S protein-ACE2 interaction (Fig. $\mathrm{S} 5 \mathrm{a}, \mathrm{b})$. For example, it remains an open question the extent to which the trimeric $\mathrm{S}$ protein can multivalently engage a bivalent ACE2 receptor. This question is of considerable importance for our understanding of how the affinity and avidity of S protein binding relate to infectivity, and what consequences this poses for therapeutic inhibition ${ }^{40,44}$. In synthetically engineered multivalent instances of the RBD-ACE2 interaction, MVsim quantitatively predicts the relative lack of steric hindrance that affords the ultra-highavidity binding observed in a study by Chan et al. ${ }^{40}$ (Fig. 5a,b). In contrast, experiments performed on more biologically mimetic S protein-ACE2 interactions indicate a significant impediment toward high avidity binding ${ }^{40}$. Using MVsim to fit therapeutic neutralization datasets reveals an effective ligand concentration, $\left[L_{e f f}\right]$, for the second engagement event between S protein and ACE2 that is 2000-fold less potent than that observed in the sterically unimpeded system (Fig. $5 \mathrm{c}, \mathrm{d}$ ) ${ }^{40}$. This inability to achieve high-avidity binding (e.g., a network in which $>95 \%$ of the populated microstates are bound with maximal valency, as is the case for the 'High' simulation in Fig. $5 \mathrm{~d}$ ) can be explained by the combination of the rigidity of the ACE2 dimer and the apparently constrained, directional motion of the linkage tethering the RBD. Quantitative modeling approaches such as these indicate a significant potential for therapeutic designs that can potently outcompete the RBD-ACE2 interaction by leveraging multivalent binding in ways inaccessible to the $S$ protein (Fig. 5e,f). Specifically, MVsim predicts that up to 1000-fold enhancements in $/ C_{50}$ values can be achieved through the use of topologically precise and constrained linkages within a designed, trivalent multispecific neutralizing therapeutic (Fig. 5). Reciprocally, MVsim further demonstrates how bivalency can be effectively leveraged with appropriate 
linkages to avidly block the RBD binding surfaces of the ACE2 dimer (Fig. S5c,d).

MVsim quantifies the dynamics of SARS-CoV-2 $S$ protein conformational switching

In addition to the sterically impeding immobility of the $S$ protein-ACE2 interaction, multivalent engagement is limited by the accessibility of the RBDs as they dynamically sample configurations ranging from the occluded yet stabilized "RBD-down" conformation to the labile yet ACE2-binding competent "RBD-up" conformation ${ }^{33,43}$. The dynamics of this range of RBD motion are a significant target of selective pressure as the benefits of maximizing host-cell binding are countered by the need to stabilize the $\mathrm{S}$ protein against spontaneous fusogenic conformational change and immune surveillance of exposed critical surfaces ${ }^{43}$. To examine intramolecular conformational changes that yield multivalent binding, we applied MVsim to simulate a multicomponent experimental system consisting of a stabilized trivalent S-protein, a set of first-order rate constants $\left(k_{u p}\right.$ and $k_{\text {down }}$ ) describing RBD conformational change, and a trivalent ligand specific for the RBDdown conformation (Fig. 6a). MVsim, constructed and parameterized in this way, succeeded not only in recapitulating the experimental multiphasic kinetic traces obtained in a study by Schoof et al. ${ }^{37}$ (Fig. 6b,c), but also in relating these response dynamics to the rates of RBD conformational switching. This modeling routine allowed MVsim to be used to extract unique parameter values for this conformational switching: $k_{u p}=0.011 \mathrm{~s}^{-}$ ${ }^{1}$ and $k_{\text {down }}=0.006 \mathrm{~s}^{-1}$ (Fig. 6d). These correspond to individual RBD half-lives of $\sim 2 \mathrm{~min}$ in the RBD-up configuration and $\sim 1 \mathrm{~min}$ in the RBD-down configuration for this stabilized, in vitro S protein system. To assess the accuracy of the MVsim-derived values of $k_{u p}$ and $k_{\text {down }}$, these rate constants were used to parameterize a simulated SPR experiment probing the lifetime of the stabilized RBD-all-up state. Here, good agreement was observed between model and experiment (Fig. 6e,f) ${ }^{37}$.

The conformational dynamics of the $S$ protein are of particular importance for understanding the mechanisms through which emerging SARS-CoV-2 mutational variants-of-concern (VOCs) increase infectiousness ${ }^{45}$. Mechanistically, S protein VOCs 
can function to stealth this protein from host immune surveillance, enhance the binding kinetics/affinity of the RBD-ACE2 interaction, stabilize the RBD-up configuration to increase the avidity of virion-host cell engagement, and/or augment conformational allostery that enables RBD binding to prime activation of membrane fusion ${ }^{45}$. To further apply MVsim to study S protein conformational dynamics, simulations were performed with the parameterized S protein RBD ensemble (Fig. S6a) to probe the effects of $k_{u p}$ and $k_{\text {down }}$ on the ensemble of RBD configurations (Fig. S6b-d) and quantitatively relate these effects to ACE2 receptor binding on host cells (Fig. S6e-g). Here, with meaningful parameterization, MVSim may aid in mechanistically parsing the often multiple virulenceenhancing features that comprise most VOCs and to quantify, for example, the consequences of a mutational profile that simultaneously alters the RBD ensemble, enhances the kinetics of ACE2 binding, and reduces the potency of a neutralizing therapeutic.

\section{Discussion}

MVsim is a new toolset created for the design, prediction, quantification, and mechanistic analysis of multivalent molecular interactions. It empowers users to simulate topologically complex multicomponent systems with an interactive GUI and to probe the relationships among configurational dynamics, cooperativity, effective concentration, and competitive binding that underlie the programmability of multivalent behavior. MVsim offers a considerable range of user inputs to parameterize the composition, kinetics, structure and topology, conformational flexibility, and component concentrations to simulate various disparate instances of multivalency in natural systems and synthetic designs.

Effectively simulating complex instances of multivalency has been hindered by the inherent combinatorial and spatial complexities that arise from binding domains sampling increasingly large, sterically constrained volumes to engage in a multitude of transient, pairwise interactions with unique energetic permissibilities ${ }^{7,8,10}$. MVsim addresses this challenge by combining rule-based modeling and multidimensional integrations to rapidly simulate system behavior by effectively tracking the evolution of hundreds of 
configurational binding state transitions throughout the lifetime of the molecular interaction. This generalized and extensible computational approach provided a means to create a consolidated modeling routine that yielded accurate and meaningful predictions of systems including simplistic beads-on-a-string topologies, intramolecular switches, and conformationally-regulated multicomponent assemblies.

Here specifically, we demonstrate the ability of MVsim to capture the unique multiphasic kinetics characteristic of multi-ligand, multispecific, and multivalent systems. We further show how MVsim can be used to predict and refine the design of systems that leverage multivalency to achieve nonlinear and ultrasensitive outputs, as well as the additional layering of multispecifity to create AND gate input/output operations. Further, we show use of MVsim in the advanced application of multispecificity toward the design of multivalent ligands capable of maximally distinguishing among pools of downstream targets and binding surfaces. Finally, to demonstrate the features and multiparameter inputs of MVsim applied to their fullest extent, a variety of therapeutically relevant structural features were computed for the SARS-CoV-2 S protein. Notably, MVsim was used to extract the effective concentration for the ACE2 interaction, quantifying the sterically unfavorable interaction that had previously been inferred from structural modeling, and to derive conformational rates of RBD switching dynamics from bulk kinetic measurements, previously quantifiable only though sophisticated single-molecule FRET experiments ${ }^{46,47}$.

The modular construction of MVsim also enables its straightforward extension to additional instances of multivalency. For example, additional configurational network tables can be applied to the source code to enable simulations of higher valencies and supramolecular topologies. Moreover, MVsim treats the calculation of effective concentration as an additional, modular mathematical step, enabling customization with any polymer end-to-end density function. Finally, the source code is further compatible with the MATLAB curve fitting toolbox to enable parameter estimation for incompletely characterized multivalent systems. 
As presented here, MVsim simulates interactions between systems of receptors and ligands with valencies of up to three. The choice of trivalent interactions was chosen to balance the number of computational steps needed to map the complete configurational network, which scales factorially with valency, with the ability to model complex and important instances of multivalency, such as those that occur in bispecific antibodies and the trimeric architecture of the SARS-CoV-2 S protein. However, the configuration nomenclature, rule-based modeling, and combinatorial computation of effective concentrations that underlie the simulations are written in the source code to accommodate all valencies and numbers of competing ligands. Moreover, further approaches can be taken to overcome the computational demands of large systems of multivalent molecules. For example, additional rules can be added to the rule-based modeling routine to allow for sparse matrix sampling of the configurational microstate network and probability density functions to significantly decrease the computational time needed to simulate more topologically and combinatorially complex interactions. Nonetheless, there is a vast number of important biological questions and biomedical problems involving bivalent or trivalent molecular interactions, and MVsim provides tools to better analyze and engineer them.

\section{Methods}

\section{MVsim}

The MVsim multivalent simulation application was built within the MATLAB app development environment (version 2021a). Our previously reported microstate network model and odds-ratio-based calculation of effective concentration served as the foundation for creating a rule-based modeling routine for the enumeration of multivalent, multispecific, and multi-ligand receptor binding states ${ }^{23}$. Multidimensional integrations of the volumetric space sampled by hinged-linked binding domains were used to enable highly resolved determinations of effective concentration that are rapidly calculable across the nanoscopic and mesoscopic molecular scales relevant to complex multivalent systems. 


\section{Experimental methods}

Multivalent and multispecific receptors were constructed using the C-terminal SH3 domain of the human adaptor protein Gads and the synthetic protein Prb, as described previously ${ }^{23}$. Multivalent and multispecific ligands incorporated the $\mathrm{SH} 3$ binding peptide (SBP) from the Gads cognate ligand SLP-76 ${ }^{48}$, as well as the synthetic designed Prbbinding DARPin, Pdar ${ }^{49}$. Association and dissociation kinetics between ligand and receptor constructs were quantified by surface plasmon resonance measurements on a Biacore S200 instrument.

\section{Documentation}

A full set of version release notes, instructions, user tips, and annotated model source code are available on the MVsim homepage at https://sarkarlab.github.io/MVsim/.

\section{Supplementary Information}

Extended methods, supplemental data and simulation figures, and MVsim source code are provided in the accompanying Supplementary Information.

\section{References}

[1] Pawson, T. Protein modules and signalling networks. Nature 373, 573-580 (1995).

[2] Hashimoto, K. \& Panchenko, A.R. Mechanisms of protein oligomerization, the critical role of insertions and deletions in maintaining different oligomeric states. Proc. Natl. Acad. Sci. USA 107, 20352-20357 (2010).

[3] Li, P. et al. Phase transitions in the assembly of multivalent signalling proteins. Nature 483, 336-340 (2012).

[4] Ditlev, J.A., Case, L.B. \& Rosen, M.K. Who's in and who's out-compositional control 
of biomolecular condensates. J. Mol. Biol. 430, 4666-4684 (2018).

[5] Kaiser, T.E., Intine, R.V. \& Dundr, M. De novo formation of a subnuclear body. Science 322, 1713-1717 (2008).

[6] Perelson, A.S. Receptor clustering on a cell surface. III. theory of receptor crosslinking by multivalent ligands: description by ligand states. Mathematical Biosciences 53, 1-39 (1981).

[7] Varner, C.T., Rosen, T., Martin, J.T. \& Kane, R.S. Recent advances in engineering polyvalent biological interactions. Biomacromolecules 16, 43-55 (2015).

[8] Mammen, M., Choi, S.K. \& Whitesides, G.M. Polyvalent interactions in biological systems: Implications for design and use of multivalent ligands and inhibitors. Angew. Chem. Int. Ed. Engl. 7, 2754-2794 (1998).

[9] Vauquelin, G. \& Charlton, S.J. Exploring avidity: understanding the potential gains in functional affinity and target residence time of bivalent and heterobivalent ligands. $\mathrm{Br}$. J. Pharmacol. 168, 1771-1785 (2013).

[10] Curk, T., Dobnikar, J. \& Frenkel, D. "Design principles for super selectivity using multivalent interactions in multivalency" in Multivalency: Concepts, Research \& Applications, Huskens, J., Prins, L.J., Haag, R., Ravoo, B.J. Eds. pp. 75-101 (Wiley, 2017).

[11] Oh, E., Akopian, D. \& Rape, M. Principles of ubiquitin-dependent signaling. Annu. Rev. Cell Dev. Biol. 34, 137-162 (2018).

[12] Kang, S.A. et al. mTORC1 phosphorylation sites encode their sensitivity to starvation and rapamycin. Science 341, 364-372 (2013).

[13] Cuesta, A.M., Sainz-Pastor, N., Bonet, J., Oliva, B. \& Alvarez-Vallina, L. Multivalent antibodies: when design surpasses evolution. Trends Biotechnol. 28, 355-362 (2010).

[14] Muller, K.M., Arndt, K.M. \& Pluckthun, A. Model and simulation of multivalent binding to fixed Ligands. Anal. Biochem. 261, 149-158 (1998).

[15] Kitov, P.I. \& Bundle, D.R. On the nature of the multivalency effect: a thermodynamic model. J. Am. Chem. Soc. 125, 16271-16284 (2003).

[16] Kane, R.S. Thermodynamics of multivalent interactions: Influence of the linker. Langmuir 26, 8636-8640 (2010).

[17] Choi, J-M., Dar, F. \& Pappu, R.V. LASSI: A lattice model for simulating phase transitions of multivalent proteins. PLoS Comput. Biol. 15, e1007028 (2019). 
[18] Martin, E.W., Holehouse, A.S., Peran, I. et al. Valence and patterning of aromatic residues determine the phase behavior of prion-like domains. Science 367, 694-699 (2020).

[19] Xu, H. \& Shaw, D.E. A simple model of multivalent adhesion and its application to influenza infection. Biophys. J. 110, 218-33 (2016).

[20] Heidenreich, M., Georgeson, J.M. at al. Designer protein assemblies with tunable phase diagrams in living cells. Nat. Chem. Biol. 16, 939-945 (2020).

[21] Masison, J., Michalski, P.J., Loew, L.M. \& Schuyler, A.D. mol2sphere: spherical decomposition of multi-domain molecules for visualization and coarse grained spatial modeling. Bioinformatics 34, 3948-3950 (2018).

[22] Michalski, P.J. \& Loew, L.M. SpringSaLaD: a spatial, particle-based biochemical simulation platform with excluded volume. Biophys. J. 110, 523-529 (2016).

[23] Errington, W.J., Bruncsics, B. \& Sarkar, C.A. Mechanisms of noncanonical binding dynamics in multivalent protein-protein interactions. Proc. Natl. Acad. Sci. USA 116, 25659-25667 (2019).

[24] Zeke, A., Lukács, M., Lim, W.A. \& Reményi, A. Scaffolds: interaction platforms for cellular signalling circuits. Trends Cell Biol. 19, 364-74 (2009).

[25] Huang, W.Y.C., Ditlev, J.A., Chiang, H.K., Rosen, M.K. \& Groves, J.T. Allosteric modulation of Grb2 recruitment to the intrinsically disordered scaffold protein, LAT, by remote site phosphorylation. J. Am. Chem. Soc. 139, 18009-18015 (2017).

[26] Brinkmanna, U. \& Kontermannb, R.E. The making of bispecific antibodies. MAbs 9, 182-212 (2017).

[27] Koenig, P-A., Das, H., Liu, H. et al. Structure-guided multivalent nanobodies block SARS-CoV-2 infection and suppress mutational escape. Science 371, eabe6230 (2021).

[28] Frey, S., Castro, A., Arsiwala, A., \& Kane, R.S. Bionanotechnology for Vaccine Design. Curr. Opin. Biotechnol. 52, 80-88 (2018).

[29] Davey, N.E. \& Morgan, D.O. Building a regulatory network with short linear sequence motifs: Lessons from the degrons of the anaphase-promoting complex. Mol. Cell 64, 12-23 (2016).

[30] MATLAB. version 9.10.0 (R2021a). Natick, Massachusetts: The MathWorks Inc. (2021).

[31] Dueber, J.E., Mirsky, E.A. \& Lim, W.A. Engineering synthetic signaling proteins with 
ultrasensitive input/output control. Nat. Biotech. 25, 660-662 (2007).

[32] Walls, A.C. et al. Structure, function, and antigenicity of the SARS-CoV-2 spike glycoprotein. Cell 181, 281-292 (2020).

[33] Huang, Y., et al. Structural and functional properties of SARS-CoV-2 spike protein: potential antivirus drug development for COVID-19. Acta Pharmacol. Sin. 41, 11411149 (2020).

[34] Lan, J. et al. Structure of the SARS-CoV-2 spike receptor-binding domain bound to the ACE2 receptor. Nature 581, 215-220 (2020).

[35] Yan, R. et al. Structural basis for the recognition of SARS-CoV-2 by full-length human ACE2. Science 367, 1444-1448 (2020).

[36] Kondo, T. et al. Antibody-like proteins that capture and neutralize SARS-CoV-2. Sci. Adv. 6, eabd3916 (2020).

[37] Schoof, M. et al. An ultrapotent synthetic nanobody neutralizes SARS-CoV-2 by stabilizing inactive Spike. Science 370, 1473-1479 (2020).

[38] Wrapp, D. et al. Structural basis for potent neutralization of betacoronaviruses by single-domain camelid antibodies. Cell 181, 1436-1441 (2020).

[39] Shiho, C. et al. Multivalent nanoparticle-based vaccines protect hamsters against SARS-CoV-2 after a single immunization. Commun. Biol. 4, 597 (2021).

[40] Chan, K.K. et al. Engineering human ACE2 to optimize binding to the spike protein of SARS coronavirus 2. Science 369, 1261-1265 (2020).

[41] Shannon, P. et al. Cytoscape: a software environment for integrated models of biomolecular interaction networks. Genome Res. 13, 2498-2504 (2003).

[42] Anthony-Gonda, K. et al. Multispecific anti-HIV duoCAR-T cells display broad in vitro antiviral activity and potent in vivo elimination of HIV-infected cells in a humanized mouse model. Sci. Transl. Med. 11, eaav5685 (2019).

[43] Li, F. et al. Structure, function, and evolution of coronavirus spike proteins. Annu. Rev. Virol. 3, 237-261 (2016).

[44] Guo, L. et al. Engineered trimeric ACE2 binds viral spike protein and locks it in "three-up" conformation to potently inhibit SARS-CoV-2 infection. Cell Res. 31, 98-100 (2021).

[45] Harvey, W.T. et al. SARS-CoV-2 variants, spike mutations and immune escape. Nat. Rev. Microbiol. 19, 409-424 (2021). 
[46] Lu, M. et al. Associating HIV-1 envelope glycoprotein structures with states on the virus observed by smFRET. Nature 568, 415-419 (2019).

[47] Lu, M. et al. Real-time conformational dynamics of SARS-CoV-2 spikes on virus particles. Cell Host Microbe 28, 880-891 (2020).

[48] Liu, Q. et al. Structural basis for specific binding of the Gads SH3 domain to an RxxK motif-containing SLP-76 peptide: a novel mode of peptide recognition. Mol. Cell 11, 471-481 (2003).

[49] Karanicolas, J. et al. A de novo protein binding pair by computational design and directed evolution. Mol. Cell 42, 250-260 (2011).

\section{Acknowledgements}

This work was supported by funding from the National Research Development and Innovation Fund (TKP2020 National Challenges Subprogram, Grant No. BME-NC) of the National Research Development and Innovation Office, the Higher Education Excellence Program (BME FIKP-BIO) of the Ministry of Human Capacities, and the Hungarian Scientific Research Fund (OTKA 119866) (to B.B.); from the National Institutes of Health (R35GM136309, R01GM113985, and R21EB022258 to C.A.S.); and from the Institute for Engineering in Medicine at the University of Minnesota (COVID-19 Rapid Response Grant to C.A.S.). The Biacore S200 instrument was made available through a shared instrument grant (S10OD021539) from the Office of Research Infrastructure Programs at the National Institutes of Health. 
bioRxiv preprint doi: https://doi.org/10.1101/2021.08.01.454686; this version posted August 2, 2021. The copyright holder for this preprint (which was not certified by peer review) is the author/funder, who has granted bioRxiv a license to display the preprint in perpetuity. It is made available under aCC-BY-NC-ND 4.0 International license.

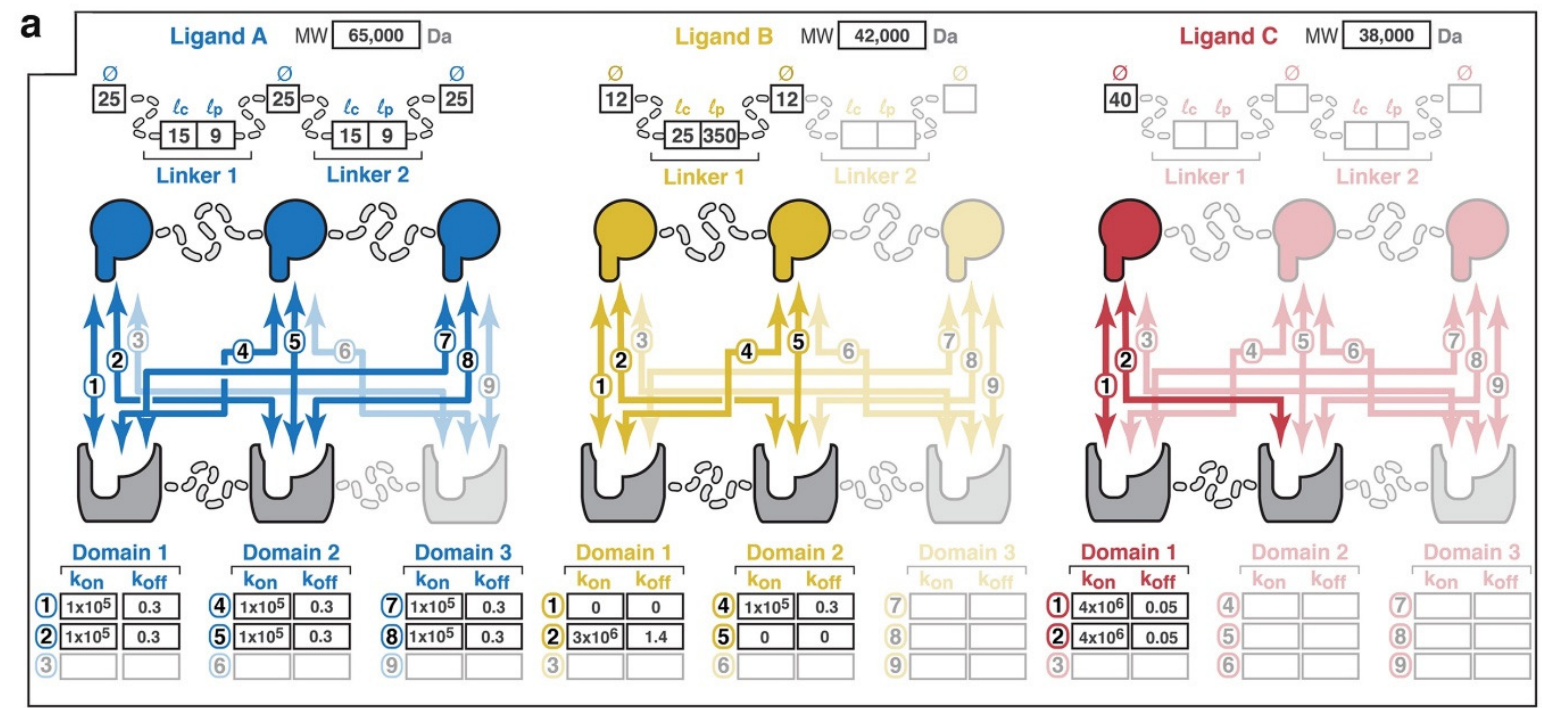

b

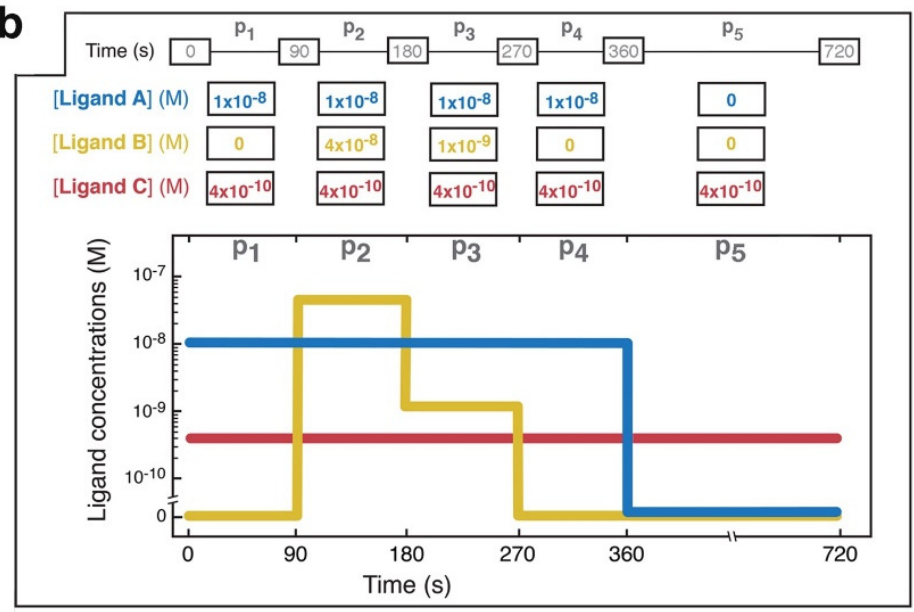

C Receptor

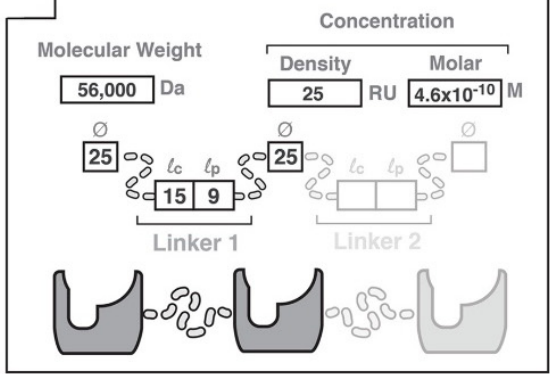

d

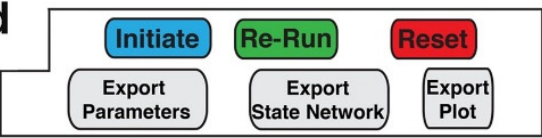

Fig. 1: The MVsim input design interface provides interactive parameter specification for systems of multivalent, multi-molecular interaction. a,b,c,d, The user specifies the design of the multivalent system of interest across individual parameterization tabs within the MATLAB app environment. (a) A point-and-click interface enables the user to select the number of ligands (up to three) and valencies of the ligand(s) and receptor (up to trivalent) that compose the multivalent system. Based upon the chosen design, the user specifies the structure of each of the ligands by entering the applicable binding domain diameters $(\varnothing)$, linker contour lengths $\left(\ell_{c}\right)$, and linker persistence lengths $\left(\ell_{p}\right)$. Further, the applicable combinatorial interactions (numbered 1 to 9 ) unique to each receptor-ligand pairing are highlighted. Tabulated parameter fields allow the user to input monovalent rate constants for each pairwise site of interaction in the multivalent system. Non-binding interactions can be indicated with $k_{\text {on }}$ and $k_{\text {off }}$ values of zero. (b) Below the kinetic input fields, a tabulated input field allows the user specify patterns of total, bulk ligand concentrations across specified points during the timecourse of the interaction. An association phase occurs during periods of nonzero bulk ligand concentration (e.g., 90-270 s for Ligand B). Dissociation phases occur when ligand is removed from the bulk solution (e.g., 360-720 s for Ligand A). Here, Ligand C is specified as continuously present in solution during the $720 \mathrm{~s}$ of the interaction timecourse. The graphical display allows visualization of the specified bulk concentration pulse pattern. Ligand pulses can be added or removed (up to 7 in total). (c) User input parameters for the receptor. Receptor concentration can be specified as either an SPR-mimicking surface density (measured in RU; where 1 RU equals $\sim 1 \mathrm{pg} / \mathrm{mm}^{2}$ ) or a molar concentration. Receptor topology is specified in the same form as described above for the ligands. (d) The MVsim controller tab enables initiation, iteration, and export of binding simulations. "Initiate" executes a user-parameterized simulation. "Re-run" executes an abbreviated and faster simulation for use where no changes were made to input parameters that alter the valency or topology of the system. "Reset" relaunches the app and clears user input parameters from all fields. 
bioRxiv preprint doi: https://doi.org/10.1101/2021.08.01.454686; this version posted August 2, 2021. The copyright holder for this preprint (which was not certified by peer review) is the author/funder, who has granted bioRxiv a license to display the preprint in perpetuity. It is made available under aCC-BY-NC-ND 4.0 International license.
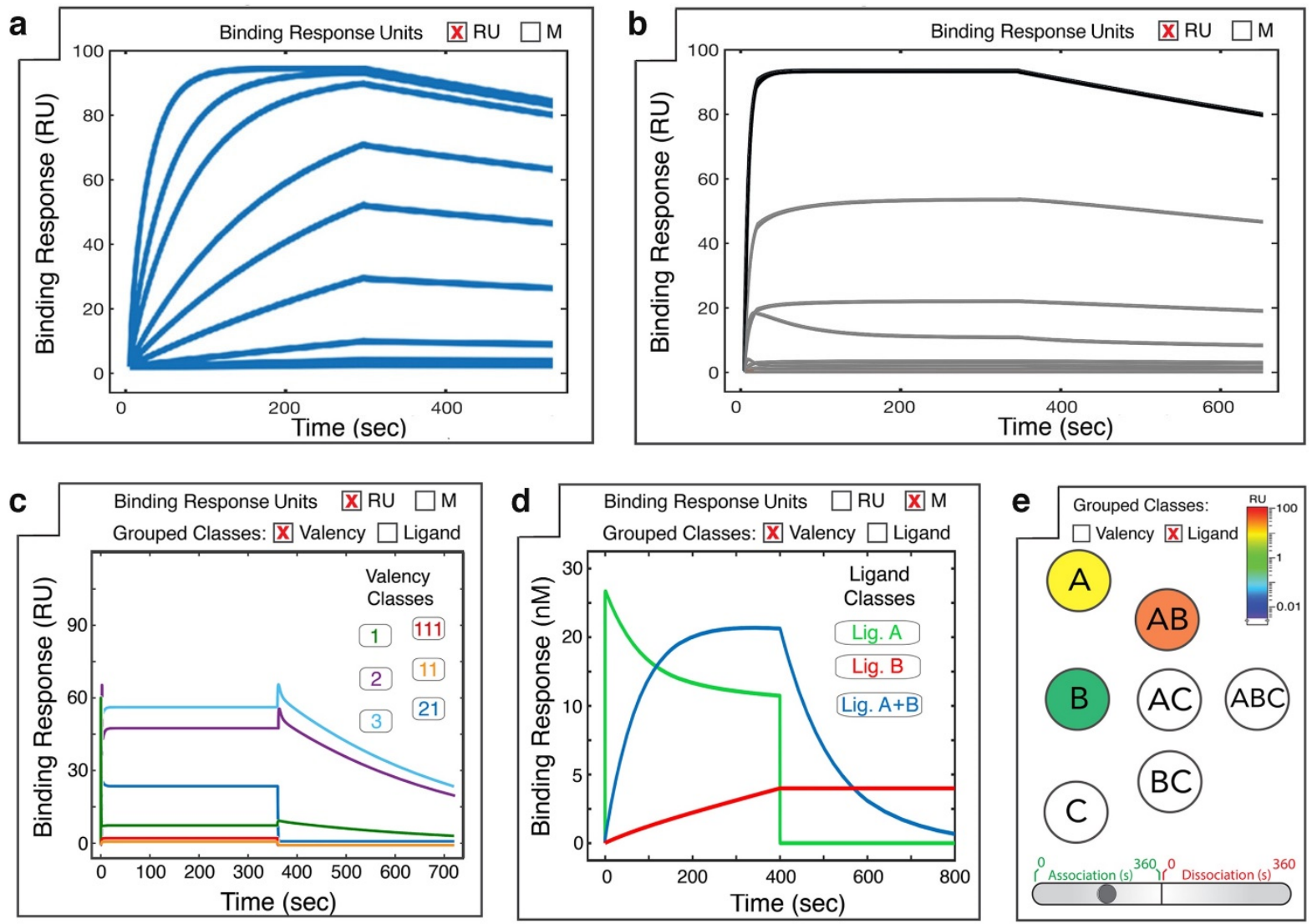

Fig. 2: MVsim generates a series of outputs that enable interactive visualization of binding responses, configurational microstates, and multi-ligand dynamics. a, A simulated SPR sensorgram displays the overall response dynamics (i.e., summation of all ligands and binding microstates) for specified ligand concentration(s). Indicated here are the binding responses for a serial dilution of a single ligand binding to a receptor with association $(0-300 \mathrm{~s})$ and dissociation (300-600 s) phases. For simple quantitative comparison between simulations, an overall effective $K_{D}$ can be calculated by the equilibrium method. b. For a specified ligand concentration, all composite microstates are displayed. c,d, To facilitate analyses of the binding responses, the microstates can be binned according to either (c) valency or (d) ligand class. e, For visual analysis of the evolution of a network of microstates in (b), an interactive graph shows population changes in microstate classes over a timecourse of association and dissociation. 
bioRxiv preprint doi: https://doi.org/10.1101/2021.08.01.454686; this version posted August 2, 2021. The copyright holder for this preprint (which was not certified by peer review) is the author/funder, who has granted bioRxiv a license to display the preprint in perpetuity. It is made available under aCC-BY-NC-ND 4.0 International license.

a

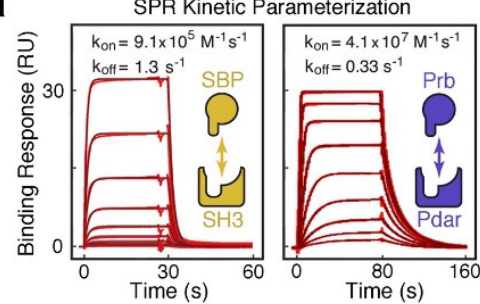

b

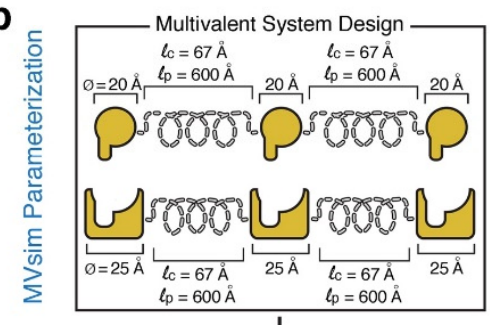

C

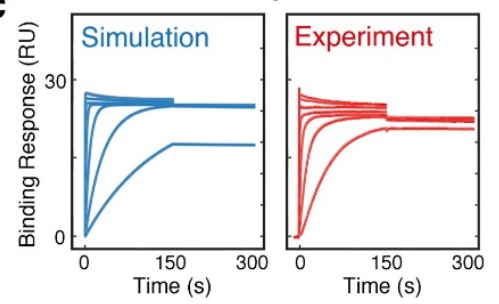

d

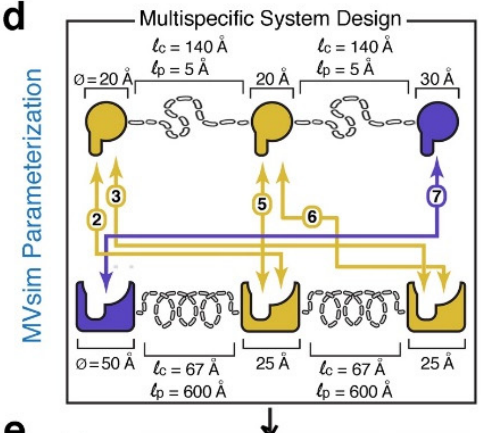

e

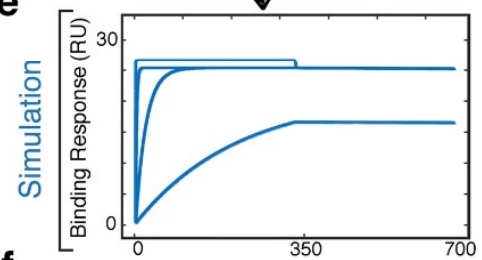

f

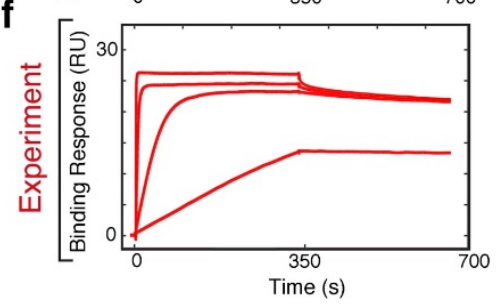

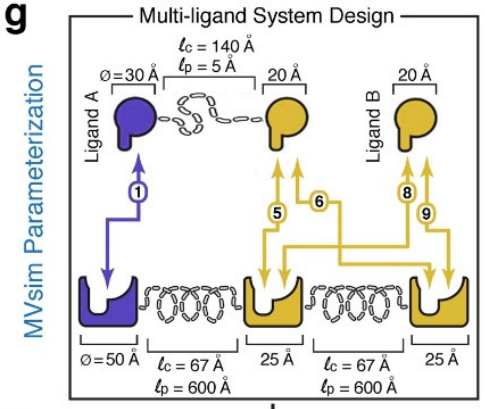

h
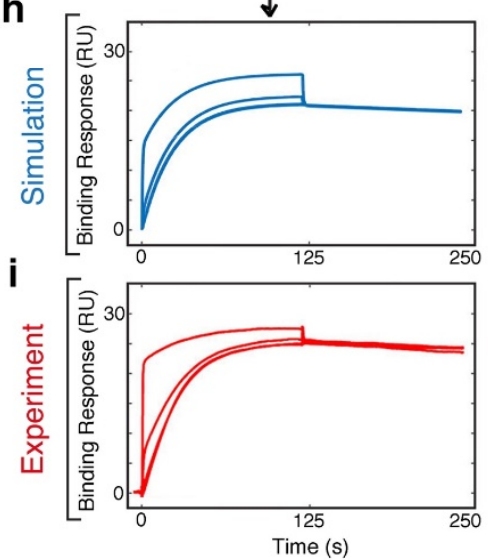

Fig. 3: MVsim accurately simulates beads-on-a-string multivalent, multispecific and multi-ligand interactions. a, Monovalent SPR kinetic rate constants were experimentally determined for the SH3-binding peptide (SBP)-SH3 (a, left panel) and Prb-Pdar (a, right panel) interactions that were used to build the multivalent systems used in this study. b, A trivalent, monospecific receptor-ligand interaction was engineered and parameterized within MVsim using values for the kinetic rate constants of association $\left(k_{o n}\right)$ and dissociation $\left(k_{\text {off }}\right)$, diameters $(\varnothing)$ for the protein-protein binding domains, and contour $\left(\ell_{c}\right)$ and persistence $\left(\ell_{p}\right)$ lengths for the alpha helical linkers. c, Simulated (c, left panel) and experimental (c, right panel) binding response dynamics for the trivalent, monospecific interaction. An overlay is shown of binding responses for six simulated ligand concentrations $(5,15,60,250,1000$, and $2000 \mathrm{nM})$. d, A trivalent, bispecific receptor-ligand interaction was engineered and parameterized within MVsim using values for the kinetic rate constants of association $\left(k_{\circ n}\right)$ and dissociation $\left(k_{\text {off }}\right)$, diameters $(\varnothing)$ for the protein-protein binding domains, and contour $\left(\ell_{c}\right)$ and persistence $\left(\ell_{p}\right)$ lengths for the alpha-helical linkers. The bidirectional arrows indicate compatible interactions between the receptor and ligand binding domains. e, Simulated binding response dynamics modeled by MVsim for the parameterized trivalent, bispecific interaction. An overlay is shown of binding responses for four simulated ligand concentrations $(5,25,100$, and $1000 \mathrm{nM})$. f, Experimental SPR binding response dynamics for the trivalent, bispecific interaction at the same four ligand concentrations as in (e). $\mathbf{g}$. The Pdar-Prb and SBP-SH3 protein-protein binding domains were used to create a multi-ligand system. $\mathbf{h}$, Simulated binding response dynamics modeled by MVsim for the parameterized dual ligand system. An overlay is shown of binding responses for three simulated mixtures of ligands $A$ and $B(1 \mathrm{nM} \mathrm{A}+2.5 \mathrm{nM} \mathrm{B} ; 1 \mathrm{nM} \mathrm{A}+50 \mathrm{nM} \mathrm{B}$; and $1 \mathrm{nM} \mathrm{A}+250 \mathrm{nM} \mathrm{B})$. i, Experimental SPR binding response dynamics for the same three dual ligand mixtures as in (h). 
bioRxiv preprint doi: https://doi.org/10.1101/2021.08.01.454686; this version posted August 2, 2021. The copyright holder for this preprint (which was not certified by peer review) is the author/funder, who has granted bioRxiv a license to display the preprint in perpetuity. It is made available under aCC-BY-NC-ND 4.0 International license.

a
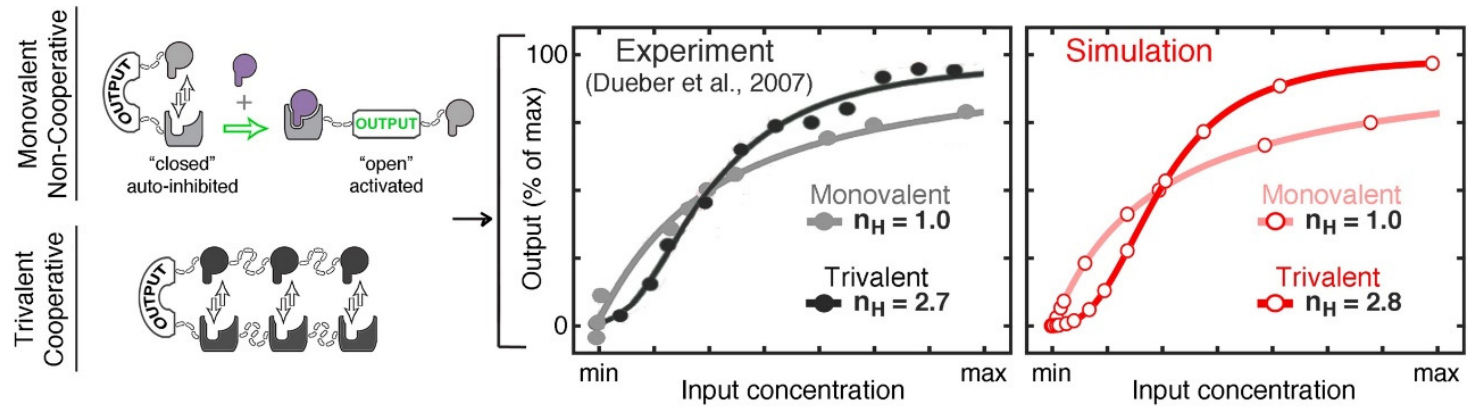

b
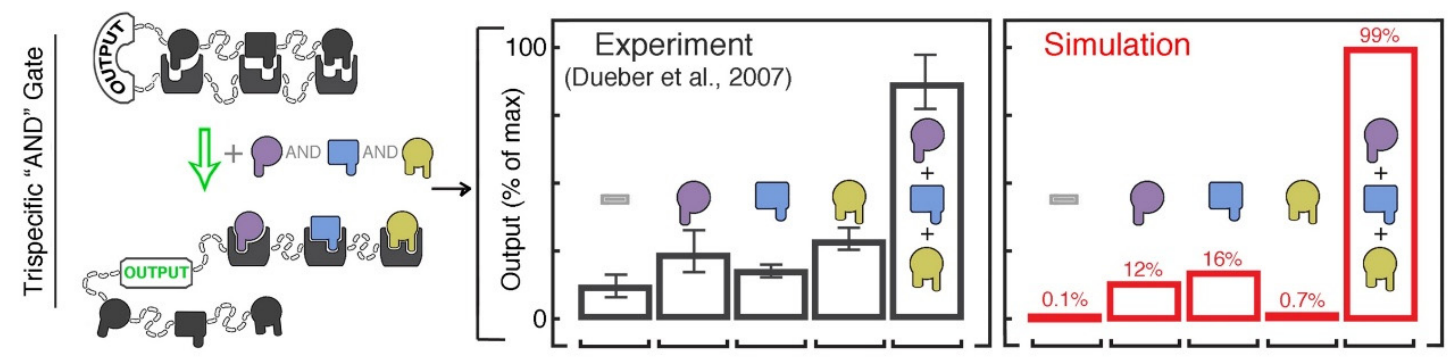

C
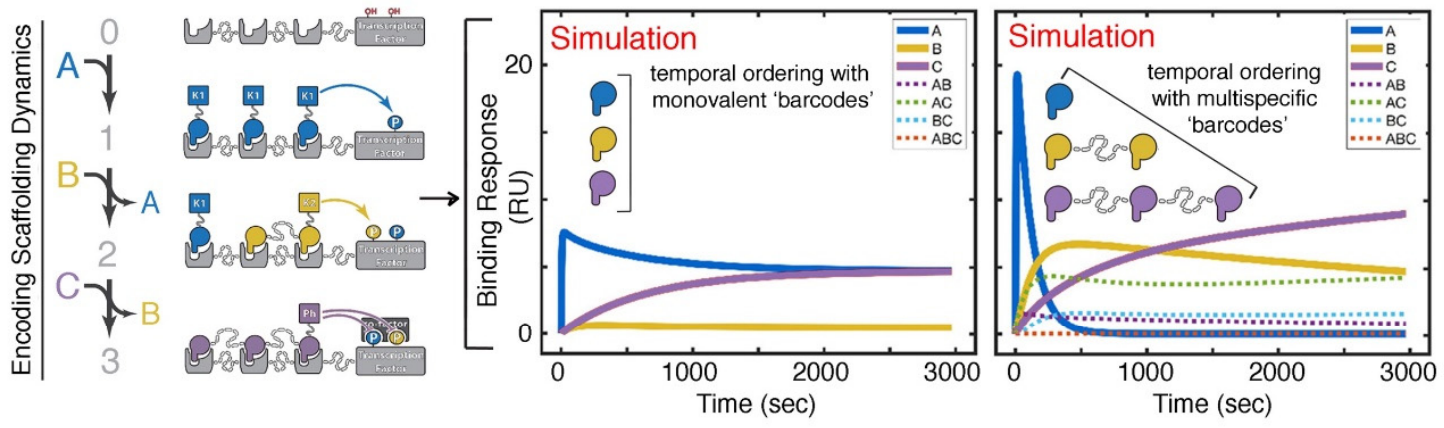

d

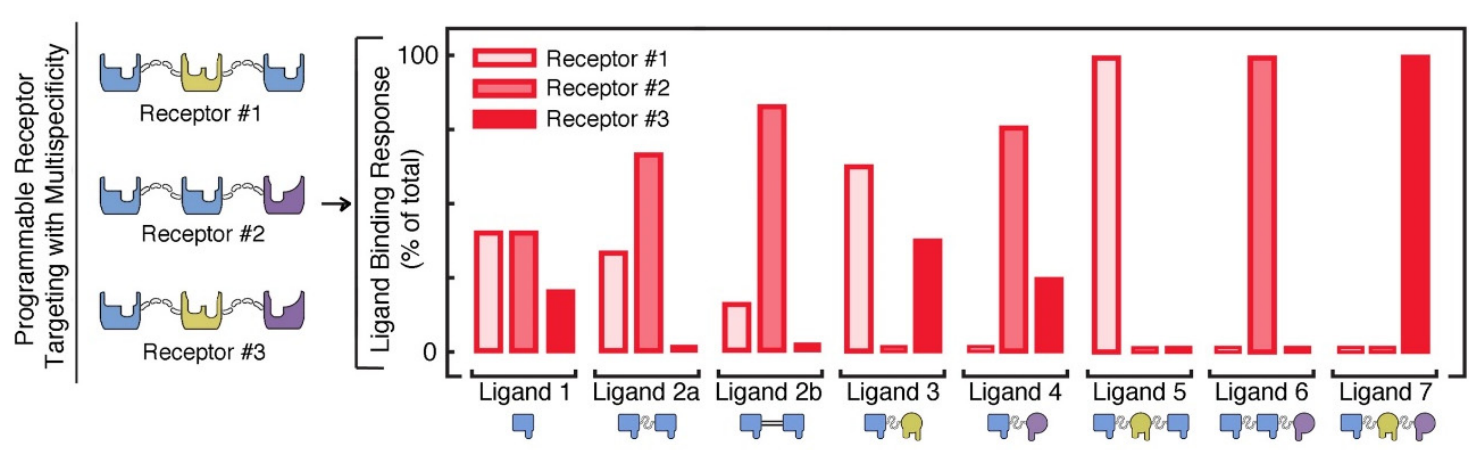

Fig. 4: MVsim predicts and informs the design of switch-like dynamics, logic operations, temporal coding, and target-receptor selectivity implicit to multivalent systems. a, Experimental response dynamics of synthetic monovalent and trivalent switches from Dueber et al. were used to benchmark the predictive performance of MVsim simulations described by the reported structural, topological, and kinetic parameters. Ultrasensitivity of each simulated response is reported with a calculated Hill coefficient $\left.\mathrm{n}_{H}\right)$ for direct comparison with the reported literature values. $\mathbf{b}$, Experimental output responses for a trispecific AND logic gate, also from Dueber et al., benchmarked against an identically parameterized system in MVsim. For clarity, the AND gate is depicted here as inline, but the actual topology necessitates consideration of twisted configurations (detailed in Fig. S4). c, Parameterization of MVsim for the design of temporally-encoded multivalent barcodes to achieve fast and sequential interactions in a model system consisting of two kinases and a phosphatase, all of which act on a signaling hub (left schematic). Here, a simulated multispecific design enables an orderly progression of binding events (right plot) that is not accessible with monovalent counterparts (left plot). d, MVsim specifies optimal design of multivalent and multispecific ligands to yield desired patterns of selective interactions within a pool of three receptors with common binding elements. 
bioRxiv preprint doi: https://doi.org/10.1101/2021.08.01.454686; this version posted August 2, 2021. The copyright holder for this preprint (which was not certified by peer review) is the author/funder, who has granted bioRxiv a license to display the preprint in perpetuity. It is made available under aCC-BY-NC-ND 4.0 International license.

Multivalent Parameterization of SARS-CoV-2 and ACE2

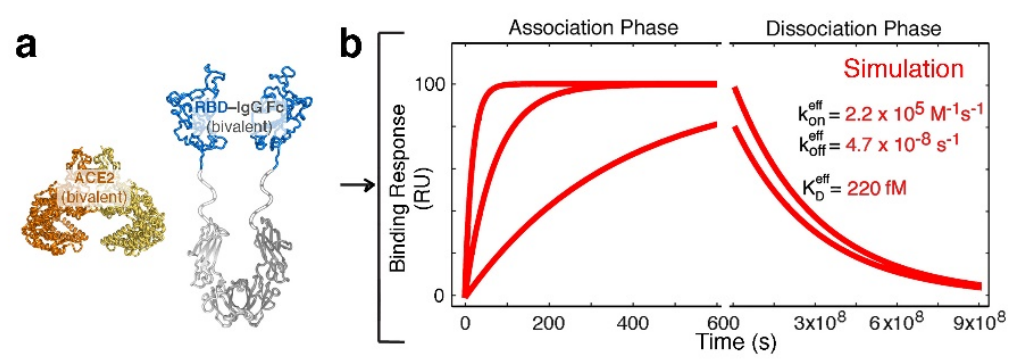

Modeling the Efficacy of a Bivalent Neutralizing Therapeutic

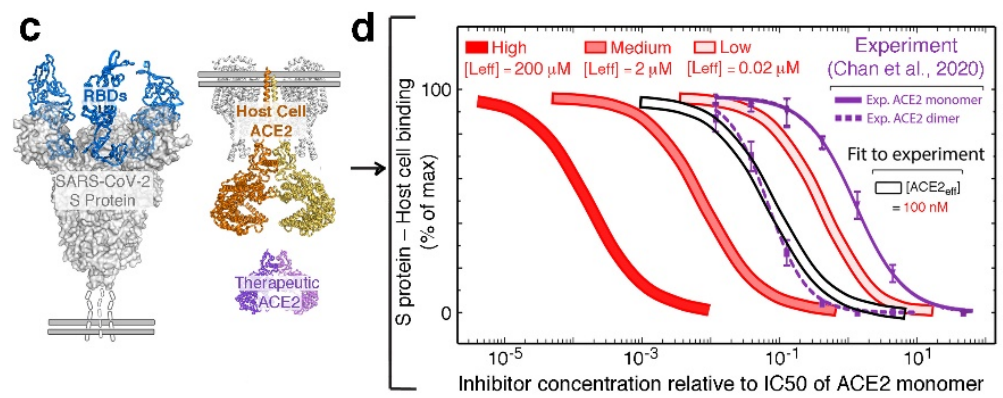

MVsim-Guided Design of SARS-CoV-2 Spike Protein Inhibitors
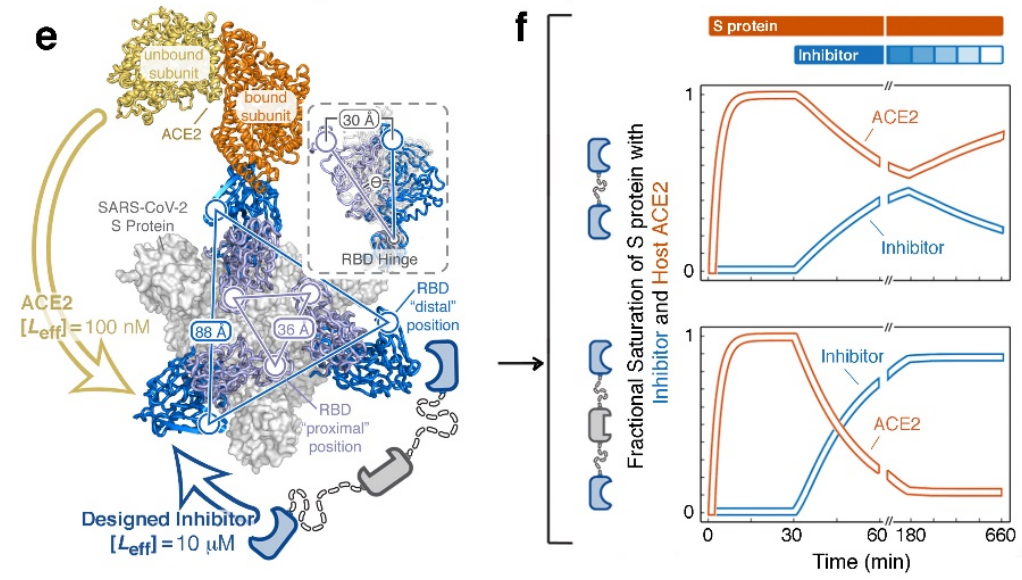

Fig. 5: MVsim models the multivalency of the SARS-CoV-2 S protein RBD and ACE2 interaction and suggests novel therapeutic design strategy. a, An idealized, flexible synthetic design of an ACE2-RBD bivalent architecture. Here, the synthetic design removes the RBD from the biologically-relevant and constrained context of the rest of the $S$ protein. $\mathbf{b}$, The flexible RBD linkers afford a high-avidity bivalent interaction with the dimeric ACE2 that was beyond the quantification limits of the experimental SPR. Here, MVsim was parameterized with the features of the experimental systems and offers prediction and quantification of the ultra-high-avidity interaction. c, Application of MVsim to a biologically-relevant instance of the SARS-CoV-2 S protein RBD and ACE2 interaction. Here, the therapeutic neutralizing activity of soluble, dimeric ACE2 (purple) was quantified in a SARS-CoV-2 pseudovirus-host-cell system ${ }^{40}$. d, The resulting $I C_{50}$ datasets were applied to $M V$ sim in order to fit for a more biologically-relevant determination of the multivalent binding capacity of the S protein-ACE2 interaction. The experimental data (purple traces) are adapted from Chan et al. ${ }^{40}$ The best fit from MVsim gave an [Leff] of $100 \mathrm{nM}$ (curve outlined in black), falling between the 'Low' and 'Medium' standard curves (shades of red), which represent no capacity and a modest steric capacity for bivalent binding, respectively. These simulations indicate that the RBDs in the full context of the $S$ protein are significantly impeded for direct bivalent binding to ACE2. e, This steric impediment can be exploited to maximize neutralizing potency by fully leveraging therapeutic multivalency. f, MVsim can test design of neutralizing inhibitors that maximally outcompete the ACE2 interaction. Designs leveraging monospecific bivalency (top panel) and trivalent bispecificity (bottom panel) are computationally modeled for their neutralizing strengths and off-rate dependent pharmacokinetic half-lives in the presence of constant S protein (orange bar above plot) and decaying concentration of inhibitor (blue bar). 
bioRxiv preprint doi: https://doi.org/10.1101/2021.08.01.454686; this version posted August 2, 2021. The copyright holder for this preprint (which was not certified by peer review) is the author/funder, who has granted bioRxiv a license to display the preprint in perpetuity. It is made available under aCC-BY-NC-ND 4.0 International license.

\section{MVsim predicts multiphasic S protein binding response dynamics}
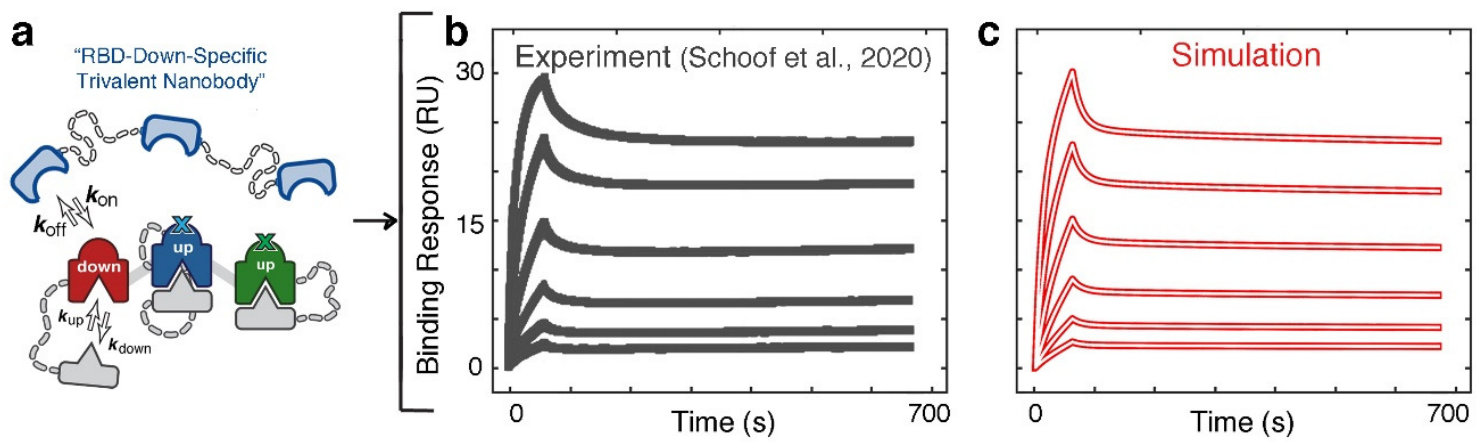

MVsim quantifies intramolecular rates of conformational S protein - RBD switching
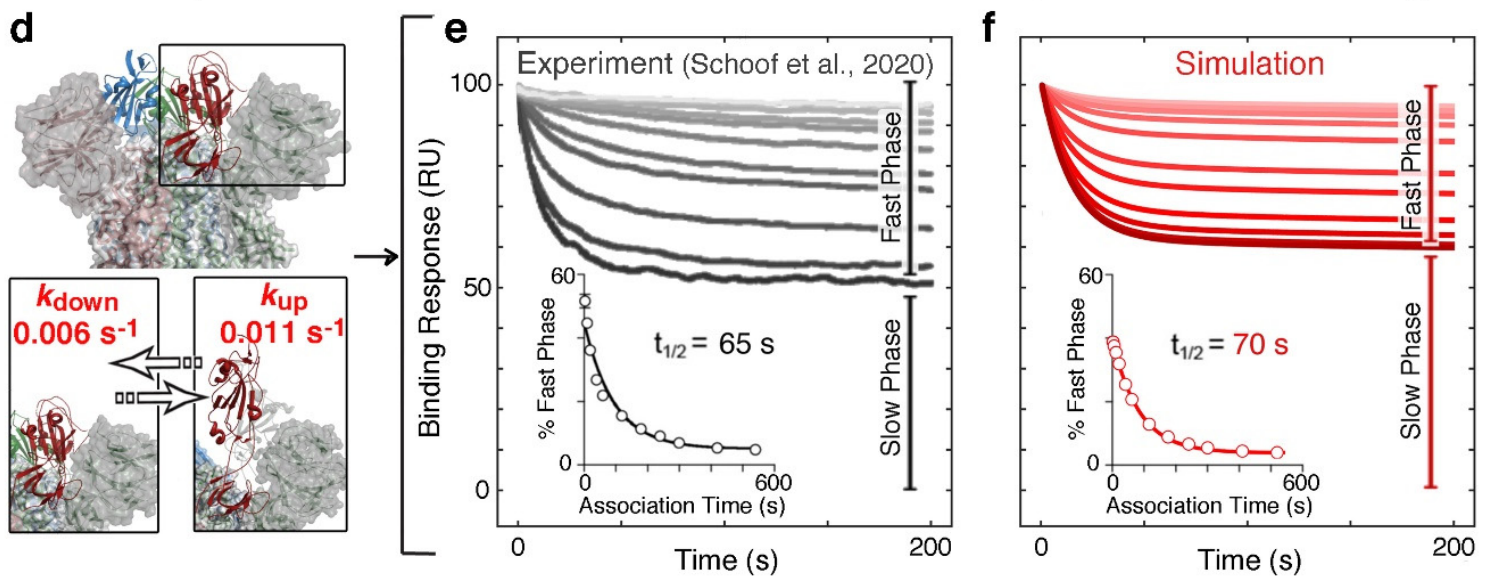

Fig. 6: MVsim quantitatively predicts multiphasic binding response dynamics of $S$ protein therapeutic neutralization and fits for rate constants of conformational switching. a, MVsim models the conformational change as an intramolecular ligand binding event (colored in grey) that toggles the trivalent S protein between "RBD-up" and "RBD-down" conformations. The conformational change is defined by a pair of first-order kinetic rate constants kup and kdown. b,c,d, The experimental kinetics of conformation-specific nanobody binding, adapted from Schoof et al. ${ }^{37}$, (b) are predicted by MVsim (c) and yield a single set of best-fit parameter values for kup and kdown for an individual RBD (d). e, Conformational switching half-life experiments, also adapted from Schoof et al. ${ }^{37}$, alter the relative proportion of "slow phase" inhibitor dissociation (i.e., high avidity bivalent and trivalent interactions) and "fast phase" inhibitor dissociation (i.e., monovalent interactions) events. Here, due to relatively slow RBD switching rates, longer association times enable more S protein to be bound in high avidity interactions, and thus give rise to small percentages of "fast phase" dissociation events. f, To assess MVsim accuracy, the fitted parameters are used in a modeling framework to simulate the experimental system and compare half-lives ( $\left.\mathrm{t}_{1 / 2}\right)$ of "RBD-up" $\mathrm{S}$ protein conformations. 